\title{
Heat exchange of a roller with a particle from polymeric material when it is pressed into tissue
}

\author{
Vladimir Fedyaev ${ }^{1, *}$, Valentin Khaliulin², Marat Faskhutdinov ${ }^{2}$, Alexey Belyaev² and Liliya \\ Sirotkina ${ }^{3}$ \\ ${ }^{1}$ IME - Subdivision of FIC KazanSC of RAS, 420111, Kazan, Russia \\ ${ }^{2}$ Kazan National Research Technical University named after A.N. Tupolev-KAI, 420111, Kazan, \\ Russia \\ ${ }^{3}$ Kazan State Energy University, 420066, Kazan, Russia
}

\begin{abstract}
We study the heat transfer of polymer particles with a roller that presses the material of the particles into the fabric. Provided that the speed of movement of the tissue with the particles relative to the roller is small, the heat exchange of the pressed particles with the environment is not taken into account, a mathematical model of conductive heat transfer in the contacting roller, polymer particle and reinforcing fabric is proposed. This model includes heat conservation equations written with respect to average temperatures of the roller, particles, fabric, as well as boundary and initial conditions. Assuming that there is perfect thermal contact between the polymer particles and the fabric, in the direction of heat propagation the average thickness of the pressed tissue particle is small, the layer of material of particles and fabric is considered thermally thin, the temperature in it varies slightly in thickness. As a result, the initial system of three equations is reduced to one equation with respect to the temperature of the roller, which is supplemented by the corresponding boundary and initial conditions. In the case when the temperature along the radius of the roller varies along its radius linearly, the specific heat flux on the surface of the roller is estimated. After that, this expression is substituted into the heat balance equation of a thermally thin layer consisting of particle material and tissue, which is integrated after certain transformations.
\end{abstract}

\section{Introduction}

In mechanical engineering, light industry, other areas of the economy, as well as in nature, processes of pressing particles from soft materials into porous layers, in particular, into fabric, are often found. It is known that the process of indentation of particles significantly depends on their mechanical properties, which, in turn, are significantly affected by the temperature of the particle material. Therefore, the assessment of the temperature of particles in contact with the tool (roller) is of practical interest [1-3].

Thus, the aim of this work is to simulate the heat transfer of an instrument (roller) with particles when they are pressed into the fabric. The following tasks are carried out:

\footnotetext{
*Corresponding author: V.L.Fedyaev@yandex.ru
} 
1. analysis of the main stages of indentation of the polymer particle material into the tissue (Fig. 1);

2. analysis of the thermal interaction of the polymer particle with the roller and fabric;

3. assessment of the dynamics of the average temperature of the material of the particles during the preload time, taking into account their deformation;

4. the solution in the engineering approximation of the mathematical problem of contact heat transfer between a roller and fabric with deformable particles located between them.
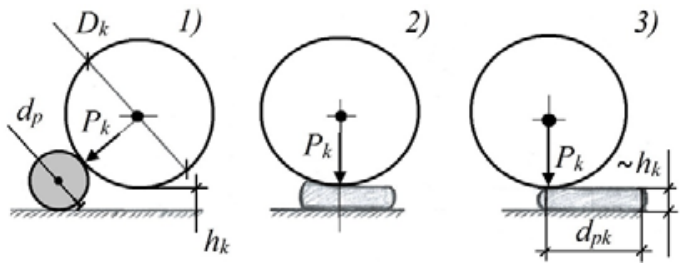

Fig. 1. The main stages of indentation of a polymer particle material into a tissue: 1 ) indentation start (time $\tau \geq 0$ ), 2) indentation at a time point $\left(\tau \square 0,5 \tau_{\mathrm{k}}\right)$, 3) indentation end $\left(\tau \leq \tau_{\mathrm{k}}\right) ; \tau_{\mathrm{k}}=d_{\mathrm{pk}} / u_{\mathrm{r}}$, $u_{\mathrm{T}}-$ the speed of tissue, particles relative to the roller

\section{Main part}

Suppose that the surface temperature of the roller $T_{\mathrm{k}}$ exceeds the temperature of the particle material $T_{\mathrm{p}}$, as a result of which it is heated. For $T_{\mathrm{k}}<T_{\mathrm{r}}$, on the contrary, the particle material cools. The thermal interaction scheme of a deformable particle and fabric with a roller is presented as follows (Figure 2). Let the compressed particle have a cylindrical shape, there is no heat transfer on the side surfaces. In the array of roller and fabric, we also select cylindrical regions of radius $\tilde{l}_{\mathrm{k}}$. It is believed that through the side surfaces their heat transfer is small. The lower surface of the fabric with a thickness of $h_{\mathrm{m}}$ is assumed to be thermally insulated; the cylinder isolated from the material of the roller extends indefinitely, in the areas of contact of the roller and fabric with the material of the particle there is an ideal thermal contact [4-9].

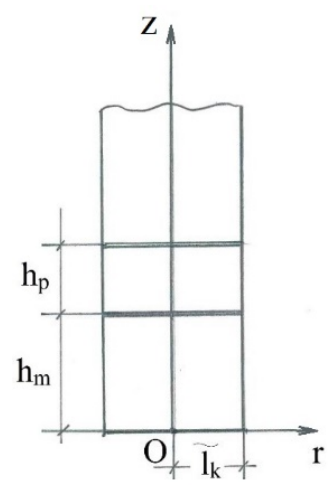

Fig. 2. Scheme of thermal interaction of a particle with a roller and fabric

Given the homogeneity of the material of the extracted parts, in the absence of internal sources, heat sinks, the heat-conjugate problem relative to the average longitudinal temperatures of the fabric material, particle, roller $T_{\mathrm{m}}, T_{\mathrm{p}}, T_{\mathrm{k}}$ in one-dimensional approximation is written as follows: 


$$
\begin{gathered}
\frac{\partial T_{\mathrm{m}}}{\partial \tau}=a_{\mathrm{m}} \frac{\partial^{2} T_{\mathrm{m}}}{\partial \mathrm{z}^{2}}\left(0 \leq \mathrm{z} \leq h_{\mathrm{m}}\right) ; \\
\frac{\partial T_{\mathrm{p}}}{\partial \tau}=a_{\mathrm{p}} \frac{\partial^{2} T_{\mathrm{p}}}{\partial \mathrm{z}^{2}}\left(h_{\mathrm{m}} \leq z \leq h_{\mathrm{m}}+h_{\mathrm{p}}\right) ; \\
\frac{\partial T_{\mathrm{k}}}{\partial \tau}=a_{\mathrm{k}} \frac{\partial^{2} T_{\mathrm{k}}}{\partial \mathrm{z}^{2}}\left(h_{\mathrm{m}}+h_{\mathrm{p}} \leq \mathrm{z}<\infty\right) ;
\end{gathered}
$$

at the boundaries of the computational domain for

$$
\begin{gathered}
\mathrm{z}=0:-\lambda_{\mathrm{m}} \partial T_{\mathrm{m}} / \partial \mathrm{z}=0 ; \\
z=h_{\mathrm{m}}:-\lambda_{\mathrm{m}} \partial T_{\mathrm{m}} / \partial \mathrm{z}=-\lambda_{\mathrm{p}} \partial T_{\mathrm{p}} / \partial \mathrm{z}, T_{\mathrm{m}}=T_{\mathrm{p}} ; \\
z=h_{\mathrm{m}}+h_{\mathrm{p}}:-\lambda_{\mathrm{p}} \partial T_{\mathrm{p}} / \partial \mathrm{z}=-\lambda_{\mathrm{k}} \partial T_{\mathrm{k}} / \partial \mathrm{z}, T_{\mathrm{p}}=T_{\mathrm{k}} ; \\
z \rightarrow \infty: T_{\mathrm{k}} \rightarrow T_{\mathrm{k} 0} ;
\end{gathered}
$$

at the initial moment of time $\tau=0$ (the beginning of particle compression)

$$
T_{\mathrm{m}}=T_{\mathrm{m} 0}, T_{\mathrm{p}}=T_{\mathrm{p} 0}, T_{\mathrm{k}}=T_{\mathrm{k} 0} \text {. }
$$

Here $a_{\mathrm{k}}=\lambda_{\mathrm{k}} /\left(\rho_{\mathrm{k}} c_{\mathrm{k}}\right) ; \lambda_{\mathrm{k}}, \rho_{\mathrm{k}}, c_{\mathrm{k}}$ - thermal conductivity coefficient, density, specific heat of the material of the roller; $T_{\mathrm{m} 0}, T_{\mathrm{p} 0}, T_{\mathrm{k} 0}$ - the initial temperature of the fabric, particles and roller; the height of the compressible particle $h_{\mathrm{p}}$ changes over time.

Considering that particles with tissue arrive in preload after radiation-convective heating, ideal thermal contact takes place between them, we replace the layer of fabric and particles with one layer with averaged thermal diffusivity

$$
\tilde{a}=\left(a_{\mathrm{m}} h_{\mathrm{m}}+a_{\mathrm{p}} h_{\mathrm{p}}\right) / h_{\mathrm{mp}} \text { and }
$$

average initial temperature

$$
\tilde{T}_{0}=\left(T_{\mathrm{m} 0} h_{\mathrm{m}}+T_{\mathrm{p} 0} h_{\mathrm{p}}\right) / h_{\mathrm{mp}}\left(h_{\mathrm{mp}}=h_{\mathrm{m}}+h_{\mathrm{p}}\right) .
$$

Due to the small height $h_{\mathrm{mp}}$ compared to the characteristic size of the roller, this layer is considered thermally thin, the temperature $\tilde{T}$ in it varies little in thickness. Entering by analogy with $\tilde{a}$ the averaged parameters

$$
\tilde{\rho}=\left(\rho_{\mathrm{m}} h_{\mathrm{m}}+\rho_{\mathrm{p}} h_{\mathrm{p}}\right) / h_{\mathrm{mp}}, \tilde{\lambda}=\left(\lambda_{\mathrm{m}} h_{\mathrm{m}}+\lambda_{\mathrm{p}} h_{\mathrm{p}}\right) / h_{\mathrm{mp}},
$$

we write the heat balance equation of the layer in question as follows:

$$
\tilde{\rho} \tilde{c} h_{\mathrm{mp}} d \tilde{T}=q_{\mathrm{k}} d \tau,
$$

where $q_{\mathrm{k}}$ is the specific heat flux entering the selected layer; the parameters $\tilde{\rho}, \tilde{c}, h_{\mathrm{mp}}$ change slowly over time (quasi-stationary approximation).

Hence, the thermal task of determining the temperature $T_{\mathrm{k}}$ will be: 


$$
\begin{gathered}
\frac{\partial T_{\mathrm{k}}}{\partial \tilde{\tau}}=\frac{\partial^{2} T_{\mathrm{k}}}{\partial^{2} \tilde{\eta}}(0 \leq \tilde{\eta} \leq 1), \\
\tilde{\eta}=0:-\tilde{\lambda}_{\mathrm{k}} \partial T_{\mathrm{k}} / \partial \tilde{\eta}=q_{\mathrm{k}}, T_{\mathrm{k}}=\tilde{T}, \\
\tilde{\eta}=1: T_{\mathrm{k}} \approx T_{\mathrm{k} 0}, \\
\tilde{\tau}=0: T_{\mathrm{k}}=T_{\mathrm{k} 0} .
\end{gathered}
$$

Here $\tilde{\eta}=\eta / \eta_{0}$ is the dimensionless vertical coordinate, the origin of which is located in the section $z=h_{\mathrm{mp}} ; \eta_{0}$ removal from the surface of the roller inward, where the temperature $T_{\mathrm{k}} \approx T_{\mathrm{k} 0} ;\left(\tilde{\tau}=a_{\mathrm{k}} \tau / \eta_{0}^{2}, \tilde{\lambda}_{\mathrm{k}}=\lambda_{\mathrm{k}} / \eta_{0}\right)$.

To obtain engineering solutions, we make further simplifications: we approximate the temperature $T_{\mathrm{k}}$ on the segment $\tilde{\eta}=(0 \div 1)$ by a linear dependence

$$
T_{\mathrm{k}} \approx \tilde{T}+\left(T_{\mathrm{k} 0}-\tilde{T}\right) \tilde{\eta},
$$

where the average temperature in the layers of tissue and particles depends on time, $\tilde{T}=\tilde{T}(\tau)$.

Having determined the specific heat flux from (6) (see (3)), substituting $q_{\mathrm{k}}$ in (1), we obtain the equation

$$
\frac{d T^{*}}{T^{*}}=-d \tau^{*}
$$

Here $T^{*}=T_{\mathrm{k} 0}-\tilde{T}, \tau^{*}=m^{*} \tau, m^{*}=\tilde{\lambda}_{\mathrm{k}} /\left(\tilde{\rho} \tilde{c} h_{\mathrm{mp}}\right)$.

The solution of equation (7) has the form

$$
T^{*}=T_{0}^{*} \exp \left(-\tau^{*}\right)
$$

where, $T_{0}^{*}=T_{\mathrm{k} 0}-\tilde{T}(\tau=0)=T_{\mathrm{k} 0}-\tilde{T}_{0}, \tilde{T}_{0}$ is the average initial temperature of the tissue layer with particles.

Formula (8) allows us to estimate the dynamics of the average temperature of the material of polymer particles during the preload time $\tau\left(\tau=0 \div \tau_{\mathrm{k}}\right)$ in the quasi-stationary approximation, taking into account the decrease in the height of the pressed particle $h_{\mathrm{p}}=h_{\mathrm{p}}(\tau)=d_{\mathrm{p}}+2 \bar{v}_{\mathrm{k}} \tau\left(1-0,5 \tau / \tau_{\mathrm{k}}\right)$, where $\left(\bar{v}_{\mathrm{k}}=u_{\mathrm{r}}\left(h_{\mathrm{k}}-d_{\mathrm{p}}\right) / d_{\mathrm{pk}}, \tau_{\mathrm{k}}=d_{\mathrm{pk}} / u_{\mathrm{r}}\right)[10-12]$.

\section{Conclusions}

Thus, in the engineering approximation, the mathematical problem of contact (conductive) heat transfer between a roller and a fabric with strongly deformable particles between them is formulated. A formula has been obtained for assessing the dynamics of heating (cooling) of particles during their pressing into the fabric, from which, in particular, it is seen that when the particles are flattened, the argument of the exponential function decreases, as a result of which the thermal effect of the roller on the temperature of the particles increases. 


\section{References}

1. V.P. Neighborhood, Properties of carbon-based construction materials (Metallurgy, Moscow, 1975)

2. E. Fitzer, Carbon fibers and composites (Mir, Moscow, 1988)

3. T.V. Komarova, Carbon composite materials (MHTI them. D.I. Mendeleev, Moscow, 1988)

4. E.R. Galimov, E.E. Tukbaev, V.L. Fedyaev, Yu.A. Pryakhin et al. High-performance technologies and equipment for the production of polymeric powder coatings (Publishing House of the Academy of Sciences of the Republic of Tatarstan, Kazan, 2016)

5. R.I. Nigmatulin, Dynamics of multiphase media (Science, Moscow, 1987)

6. V.P. Isachenko, V.A. Osipova, A.S. Sukomel, Heat transfer (Higher School, Moscow, 1988)

7. S.S. Kutateladze, Heat transfer and hydrodynamics (Energoatomizdat, Moscow, 1990)

8. K.O. Bennett, D.E. Myers, Hydrodynamics, heat transfer and mass transfer (Nedra, Moscow, 1966)

9. V.N. Lukanina, Heat engineering (Higher School, Moscow, 2000)

10. A.A. Ilyushin, Continuum mechanics (Moscow State University Press, Moscow, 1978)

11. N.I. Bezukhov, Fundamentals of the theory of elasticity, plasticity and creep (Higher School, 1968)

12. I.K. Suvorov, Metal forming (Higher School, Moscow, 1980) 